\title{
GAwI: A Comprehensive Workspace Awareness Library for Collaborative Web Applications
}

\author{
Matthias Heinrich ${ }^{1}$, Franz Josef Grüneberger ${ }^{1}$, Thomas Springer ${ }^{2}$, \\ Philipp Hauer ${ }^{3}$, and Martin Gaedke ${ }^{3}$ \\ 1 SAP AG, Germany \\ \{matthias.heinrich, franz . josef .grueneberger\}@sap.com \\ 2 Dresden University of Technology, Germany \\ thomas.springer@tu-dresden.de \\ 3 Chemnitz University of Technology, Germany \\ \{philipp.hauer, martin.gaedke\}@cs.tu-chemnitz.de
}

\begin{abstract}
In the light of the Web 2.0 movement, the rise of collaborative web applications like Google Docs lead to an enormous end-user adoption largely due to their advanced multi-user capabilities (i.e. document synchronization in real-time and sophisticated workspace awareness support). Nevertheless, the development of collaborative web applications, in particular, the implementation of workspace awareness widgets such as telepointers, radar views, etc., is costly since there are no comprehensive libraries promoting widget reuse. Therefore, we introduce the enhanced Generic Awareness Infrastructure (GAwI) allowing for an efficient development of collaborative web applications. Efficiency is fostered through GAwI's reusable set of widgets and its non-invasive integration. In this paper, we expose GAwI's enhanced widget set, verify GAwI's comprehensiveness in terms of workspace awareness and demo the GAwI integration and GAwI widgets in two widespread open-source editors.
\end{abstract}

\section{Introduction}

Collaborative web applications like Google Docs allow multiple users to change the very same document simultaneously. Besides document synchronization and conflict resolution, collaborative real-time applications require a third distinctive multi-user capability, namely, workspace awareness, which is commonly exposed through awareness widgets such as participant lists, telepointers, radar views, etc. In essence, Workspace Awareness (WA) supports collaborators to understand the actions and intentions of others 1]. For example, participant lists allow understanding who is in the shared workspace or creation coloring widgets indicate who authored new content.

Nevertheless, even though collaborative web applications necessitate WA capabilities to efficiently support joint work [2], modern collaboration frameworks targeting the web (e.g. Apache Wave [3], beWeeVee [4] or CEFX [5]) do not offer WA features at all. Consequently, the traditional from-scratch implementation of WA functionality for collaborative web applications results in time-consuming and costly development projects neglecting WA widget reuse.

F. Daniel, P. Dolog, and Q. Li (Eds.): ICWE 2013, LNCS 7977, pp. 482-485, 2013.

(C) Springer-Verlag Berlin Heidelberg 2013 
Therefore, we introduced the Generic Awareness Infrastructure (GAwI) in [6] advocating non-invasive WA incorporation and WA widget reuse. In this paper, we present an enhanced set of reusable awareness widgets including a telepointer, radar view, artifact marking and telecaret widget. Moreover, we validate the comprehensiveness of the resulting WA library and showcase the enhanced awareness widget set in a dedicated screencast that is available at http://vsr.informatik.tu-chemnitz.de/demo/GAwI/

\section{GAwI Overview}

The collaboration system architecture including GAwI components is shown in Figure 1(a) and consists of a server and an arbitrary number of clients. In detail, the depicted collaboration system comprises the abstract web editor stack (including Editor UI, Editor API, W3C APIs and the DOM), the concurrency control system (including the Generic Sync Adapter and the server-side DOM Sync Service) and the Generic Awareness Infrastructure. While the generic concurrency control, which is discussed in [7, synchronizes DOM changes and resolves editing conflicts, GAwI captures, distributes and provides input for WA widgets [6]. The generic nature of GAwI is promoted by relying exclusively on standardized W3C APIs instead of editor-specific ones that would entail an extra WA adapter implementation for each supported web editor.

Nevertheless, the GAwI presented in [6] accommodates only two awareness widgets (participant list and creation coloring) and thus, lacks WA support in a comprehensive and holistic manner. Gutwin et al. introduced the 10 WA elements 22 listed in Figure 1(b) (presence, identity, etc.) that have to be covered by a comprehensive WA library. Currently, the spider chart in Figure 1(b) gives a rough estimation to what extent WA elements are covered by GAwI [6]. On the one side, the participant list reflects if collaborators are present and reveals their identity; on the other hand, the creation coloring widget discloses who carried out what action and which artifacts were affected. In particular, the where-elements location, gaze, view and reach are not sufficiently supported.

\section{GAwI Enhancements}

To enhance GAwI's existing set of WA widgets and to effectively support all 10 WA elements, we implemented 4 extra awareness widgets depicted in Figure 1(c). In the following, we discuss implementation details that allow for reuse.

Telepointer: To be aware of the mouse cursor of other participants, the telepointer mimics the cursor movements remotely. Our implementation built on top of standardized W3C APIs starts capturing local mouse cursor changes when the DOM Core mousemove event is fired. Since participants' viewports may differ in various aspects (e.g. size, zoom level or resolution), the $\mathrm{x}$ and $\mathrm{y}$ window coordinates cannot be exploited. Instead, we leverage the underlying DOM node (e.g. a text node representing a heading or an SVG node visualizing a circle) as 


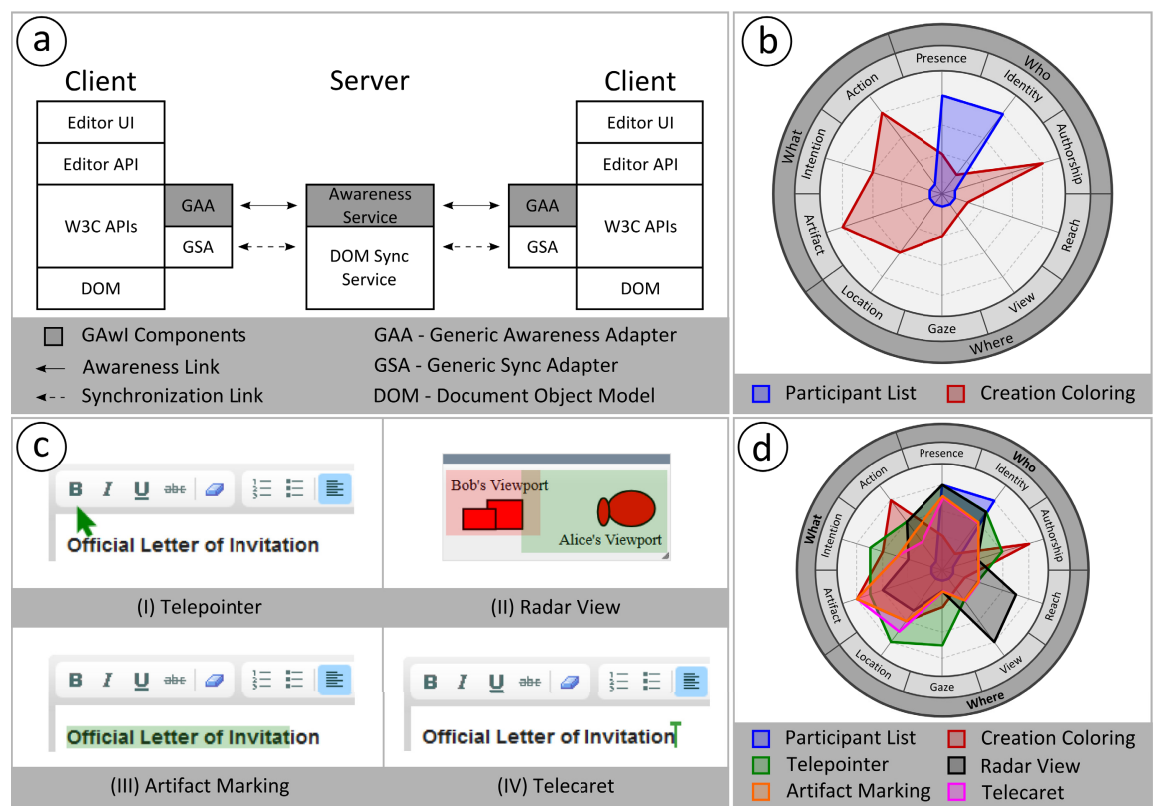

Fig. 1. (a) The GAwI architecture [6] (b) WA elements 2] and their as-is coverage (c) Set of added awareness widgets (d) Comprehensiveness analysis of WA support

the reference point for positioning calculations which results in more accurate positions in heterogeneous environments. The telepointer shape is drawn on a HTML5 canvas layer that spans the entire shared workspace. Adopting a pixelbased canvas is essential since SVG-based solutions may impair performance.

Radar View: To highlight where participants are working, the radar view exposes a miniaturized view including all document artifacts and semi-transparent viewports. Miniaturizing HTML documents is not trivial since HTML consists of a variety of different media objects that cannot be scaled in a uniform manner (e.g. scaling fonts differs from scaling images). Hence, we used the html2canvas JavaScript library to generate a pixel-based representation from the DOM view that can be uniformly scaled to a miniature view. However, taking a snapshot of the DOM view is costly and thus, the radar view is only updated if a fixed time interval elapsed and not if document artifacts are changed.

Artifact Marking: Local artifact selections are highlighted remotely by the artifact marking widget and thus provide means to focus the attention of collaborators on a specific object. The capturing phase is triggered by mouse events (e.g. click) and has to ensure that the selection area is properly calculated. Absolute coordinates are once again not an option due to heterogeneous window sizes, zoom levels, etc. The HTML5 Editing API provides so called Range objects that allow specifying continuous selection parts based on content rather than on coordinates. These range objects are exploited to draw a properly dimensioned, semi-transparent $\langle$ div $\rangle$ node on top of selected document artifacts. 
Telecaret: Communicating the local text cursor position to teammates is the task of the telecaret. Thereby, DOM keyboard events (e.g. keydown) are used to initiate the telecaret re-positioning. Analog to calculating artifact marking areas, the range object also enables telecaret positioning whereas range objects with the same start and end point are adopted. The visualization is materialized through an extra <div> element inheriting the participant list's color code.

Besides providing details about the application-agnostic implementation, Figure 1(d) depicts an assessment of the individual widgets regarding their support for the $10 \mathrm{WA}$ elements. Even though this expert estimate is coarse-grained and an end-user study could detail the results, the trend becomes apparent that the sum of all widgets can comprehensively cover all WA elements. However, the support for gaze, reach and intention leaves room for improvement.

\section{GAwI Demonstration and Conclusions}

In this demo (cf. http://vsr.informatik.tu-chemnitz.de/demo/GAwI/), we leverage two web-based open-source editors, namely, CKEditor [8] and SVGedit [9], to showcase GAwI capabilities. First, we show the non-invasive integration of GAwI in the prominent CKEditor, i.e. the integration entails no source code changes to the editor's JavaScript code. Second, we demo WA widgets in a word processor application (CKEditor) and third, we employ a graphics application (SVG-edit) to again present the generic WA widgets.

In essence, in this paper, we discussed a set of AW widgets that drive development efficiency for collaborative web applications due to their generic nature and non-invasive incorporation into existing web applications. In a next step, we will conduct a user study adopting the collaborative CKEditor and the multi-user SVG-edit to thoroughly assess the functionality and usability of GAwI widgets.

Acknowledgments. This work was partially supported by funds from the European Commission (project OMELETTE, contract number 257635).

\section{References}

1. Dourish, P., Bellotti, V.: Awareness and Coordination in Shared Workspaces. In: CSCW, pp. 107-114 (1992)

2. Gutwin, C., Greenberg, S.: A Descriptive Framework of Workspace Awareness for Real-Time Groupware. Computer Supported Cooperative Work 11, 411-446 (2002)

3. ASF: Apache Wave, http://incubator .apache.org/wave/ (2013)

4. Corvalius: beWeeVee, http://www.beweevee.com(2013)

5. Gerlicher, A.: CEFX (2013), http://sourceforge.net/projects/cefx/

6. Heinrich, M., Grüneberger, F.J., Springer, T., Gaedke, M.: Reusable Awareness Widgets for Collaborative Web Applications - A Non-invasive Approach. In: Brambilla, M., Tokuda, T., Tolksdorf, R. (eds.) ICWE 2012. LNCS, vol. 7387, pp. 1-15. Springer, Heidelberg (2012)

7. Heinrich, M., Lehmann, F., Springer, T., Gaedke, M.: Exploiting Single-User Web Applications for Shared Editing: A Generic Transformation Approach. In: WWW, pp. 1057-1066 (2012)

8. CKSource: CKEditor (2013), http://ckeditor.com/

9. Schiller, J., Rusnak, P.: SVG-edit (2013), http://code.google.com/p/svg-edit/ 